\title{
Paediatric focal intracranial suppurative infection: a UK single-centre retrospective cohort study
}

Fabian J. S. van der Velden ${ }^{1,2}$, Alexandra Battersby ${ }^{1}$, Lucia Pareja-Cebrian ${ }^{3}$, Nicholas Ross ${ }^{4}$, Stephen L. Ball ${ }^{5}$ and Marieke Emonts ${ }^{1,6^{*}}$ id

\begin{abstract}
Background: Paediatric focal intracranial suppurative infections are uncommon but cause significant mortality and morbidity. There are no uniform guidelines regarding antibiotic treatment. This study reviewed management in a tertiary healthcare centre in the United Kingdom and considers suggestions for empirical treatment.

Methods: A retrospective, single-centre cohort review of 95 children ( $<18$ years of age) with focal intracranial suppurative infection admitted between January 2001 and June 2016 in Newcastle upon Tyne, United Kingdom. Microbiological profiles and empirical antibiotic regimens were analysed for coverage, administration and duration of use. Mortality and neurological morbidity were reviewed. Data was analysed using t-tests, Mann-Whitney $U$ tests, independent-samples median tests, and $x^{2}$-tests where appropriate. $P$-values $<0.05$ were considered statistically significant.
\end{abstract}

Results: Estimated annual incidence was 8.79 per million. Age was bimodally distributed. Predisposing factors were identified in $90.5 \%$, most commonly sinusitis $(42.1 \%)$ and meningitis (23.2\%). Sinusitis was associated with older children $(p<0.001)$ and meningitis with younger children $(p<0.001)$. The classic triad was present in 14.0\%. $43.8 \%$ of 114 isolates were Streptococcus spp., most commonly Streptococcus milleri group organisms. Twelve patients cultured anaerobes.

Thirty one empirical antibiotic regimens were used, most often a third-generation cephalosporin plus metronidazole and amoxicillin (32.2\%). 90.5\% would have sufficient cover with a third generation cephalosporin plus metronidazole. $66.3 \%$ converted to oral antibiotics. Median total antibiotic treatment duration was 90 days (interquartile range, $60-115.50$ days).

Mortality was 3.2, 38.5\% had short-term and 24.2\% long-term neurological sequelae.

Conclusions: Paediatric focal intracranial suppurative infection has a higher regional incidence than predicted from national estimates and still causes significant mortality and morbidity. We recommend a third-generation cephalosporin plus metronidazole as first-choice empirical treatment. In infants with negative anaerobic cultures metronidazole may be discontinued.

Keywords: Paediatrics, Brain abscess, Empyema, subdural, Antimicrobials

\footnotetext{
* Correspondence: marieke.emonts@ncl.ac.uk

${ }^{1}$ Paediatric Immunology, Infectious Diseases and Allergy Department,

Newcastle upon Tyne Hospitals NHS Foundation Trust, Great North Children's

Hospital, Newcastle upon Tyne NE1 4LP, UK

${ }^{6}$ Institute of Cellular Medicine, Newcastle University, Newcastle upon Tyne

NE2 $4 \mathrm{HH}$, UK

Full list of author information is available at the end of the article
}

(c) The Author(s). 2019 Open Access This article is distributed under the terms of the Creative Commons Attribution 4.0 International License (http://creativecommons.org/licenses/by/4.0/), which permits unrestricted use, distribution, and reproduction in any medium, provided you give appropriate credit to the original author(s) and the source, provide a link to the Creative Commons license, and indicate if changes were made. The Creative Commons Public Domain Dedication waiver (http://creativecommons.org/publicdomain/zero/1.0/) applies to the data made available in this article, unless otherwise stated. 


\section{Introduction}

Focal intracranial suppurative infections are serious conditions rarely seen in children. [1-5] They are divided in three categories: brain abscess (BA), subdural empyema $(\mathrm{SDE})$, and extradural empyema (EDE). In the pre-antibiotic era, mortality reached nearly $100 \%$ [6], decreasing to around $36-60 \%$ in the 1970 s when antibiotics became more readily available. $[3,7]$ Since then mortality has dropped further, ranging $3.7-24 \%$, as computed tomography was introduced and cranial imaging improved, and metronidazole became part of most standard empirical regimens in the 1980s. [7-14]

Although mortality has dropped, these infections continue to cause significant mortality and morbidity, and can lead to rapid patient deterioration, thus adequate diagnostics and early aggressive treatment are crucial to optimise chances of complete recovery. $[4,9,10,14-16]$

The annual estimated incidence is $4-5.3 / 1,000,000$ [2, 12], with higher incidences in developing countries. [15, 17] For the United Kingdom (UK) this translates to approximately 3 paediatric cases per tertiary healthcare centre annually.

In 74.4-91.6\% a predisposing factor can be determined. [7, 14, 18] Compared to adults, congenital heart disease (CHD) and immunosuppression occur more frequently in children. [2] Other common predispositions in children are meningitis and sinusitis. $[4,9,16,17] \mathrm{Pa}$ tients can present with a variety of symptoms. The classic triad consisting of headache, fever, and focal neurological deficits is reported in $8.4-20 \%$ of children. $[3,12,14,18]$ Treatment is multidisciplinary consisting of antibiotics and neurosurgery. [3, 5] Neurosurgical intervention aids pathogen identification, reduces lesion size and decompresses, aiming to reduce effects on surrounding structures. [19]

Several microorganisms have been implicated, but most commonly cultured are Streptococci and Staphylococci. [1, 2, 10, 12, 14, 16, 20-22] 11.1-33\% of patients grow anaerobic species $[3,7,12]$, and $14.3-27 \%$ of BAs is polymicrobial. [11, 14] Empirical antibiotic treatment is broad-spectrum to cover these organisms, and rationalised on microbiological guidance.

There is no standardised empirical antibiotic treatment. $[9,16,23,24]$ Often first-choice empirical treatment is a third-generation cephalosporin plus metronidazole. [12, 19] These antibiotics have adequate pharmacokinetics and pharmacodynamics to achieve therapeutic concentrations within the central nervous system. [25]

In the UK there are currently no national guidelines regarding treatment of paediatric suppurative intracranial infections. [12] Internationally proposed consensus documents and guidelines are controversial [19, 23, 24, 26], because they are based on combined adult and paediatric literature. Paediatric evidence remains sparse, mainly consisting of case-series with small patient populations. $[3,11,18,21]$

Published guidelines suggest antibiotic treatment for 6-8 weeks by intravenous administration only. [24] However, guidelines from The Infection in Neurosurgery Working Party of the British Society for Antimicrobial Chemotherapy [27] recommend 1-2 weeks of intravenous administration and to consider conversion to oral administration, depending on clinical response and decreasing $\mathrm{C}$-reactive protein (CRP), to complete the antibiotic course.

This study evaluated local management of paediatric focal intracranial suppuration and reviewed antibiotic practice in order to consider empirical antibiotic guidelines.

\section{Methods}

This single-centre retrospective cohort study reviewed paediatric patients with $\mathrm{BA}, \mathrm{SDE}$, and $\mathrm{EDE}$, admitted to the Great North Children's Hospital $(\mathrm{GNCH})$, a tertiary healthcare centre for paediatric infectious diseases and neurosurgery in the North East of England between January 2001 and June 2016. Local Caldicott approval was obtained.

\section{Patient identification}

Eligible cases were identified by assessing the paediatric infectious diseases, intensive care (PICU) and neurosurgery records, the previously described local cohort [9], and the hospital clinical coding database.

The following World Health Organization International Classification of Disease Codes [28], tenth revision, were used: G06.0 Intracranial abscess and granuloma, G06.2 Extradural and subdural abscess, unspecified, and G07 Intracranial and intraspinal abscess and granuloma in diseases classified elsewhere.

Cases were included if the patient was $<18$ years of age on admission, had a confirmed diagnosis according to clinical information, and had diagnosis confirmation by radiology.

\section{Data collection}

Data was collected from medical records and electronic systems used within the hospital. Data was recorded on patient demographics, admission duration, confirmed diagnosis, presenting symptoms, symptom duration before admission, predisposing factors, laboratory and microbiology results, antibiotics, neurosurgical management, morbidity and mortality.

\section{Microbiology}

Microbiological data was collected from cultures taken during admission. This included blood, cerebrospinal 
fluid (CSF), intracranial pus and paranasal sinus cultures.

Positive cultures were defined as cultures with microorganism growth or positive polymerase chain-reaction (PCR) (PCR panel: Streptococcus spp., Neisseria meningitides, Fusobacterium spp., Aspergillus spp. Staphylococcus spp). Antibiotic resistance patterns were recorded.

Cases without growth in any cultures, had cultures reassessed. As per microbiology protocol, gram stains of every sample are examined microscopically before culturing and sent for PCR identification if no growth is observed after incubation. If either test was found positive, the culture was considered positive. Otherwise, the culture was considered negative. Due to changes in the microbiology protocol between 2001 and 2016, not all samples were sent for PCR in growth-negative cases owing to increasing availability of PCR since 2004 only.

\section{Statistical analysis}

Data was analysed with SPSS $^{\circ}$ Statistics, version 22 (IBM Corporation, Armonk, New York). Normality was assessed with the Shapiro-Wilk test. Normally distributed data was analysed with unpaired t-tests or one-sample t-tests. Not normally distributed data was analysed with Mann-Whitney $U$ tests and independent-samples median tests. $X^{2}$-tests were used where appropriate. $P$-values $<0.05$ were considered statistically significant.

\section{Results}

107 eligible cases were identified. 7 cases were excluded due to incorrect coding, case notes were unavailable for 3 , and 2 patients were primarily treated in other healthcare centres. 95 cases were suitable for analysis, of which 2 had chronic granulomatous disease (CGD) whose data were only suitable for diagnosis, predisposing factors and mortality analysis (Fig. 1).

This centre treated on average 6.13 cases annually. All patients originated from the $\mathrm{GNCH}$ catchment area. The child population (aged 0-19 years) of this area was 697,200 in 2014 . [29, 30] This leads to an estimated annual incidence of $8.79 / 1,000,000$.

\section{Patient demographics}

There were 60 males (63.2\%). Median admission age was 10.21 years (interquartile range (IQR), $1.57-12.67$ years) and was bimodally distributed (Figure 2). Age at presentation was not significantly different between males and females (median, 8.73 and 11.53 years, respectively, $p=0.172$ ). There was no association between age or sex and type of intracranial suppurative infection.

\section{Diagnosis and localisation}

All patients underwent cranial imaging with contrast. 33/95 patients had BA (34.7\%), 46/95 had SDE (48.4\%),
2/95 had EDE (2.2\%), 6/95 had BA and SDE (6.3\%), 1/95 had BA and EDE (1.1\%), and 7/95 had SDE and EDE (7.4\%). 13/40 (32.5\%) had multiple abscesses. The majority of brain abscesses was located in the frontal lobe (50\%).

\section{Predisposing factors}

Predisposing factors were identified in 86 patients (90.5\%). Most common were sinusitis (42.1\%) and meningitis (23.2\%) (Table 1). $27 / 40$ sinusitis patients were in the $10-15$ years age group and 16/22 meningitis patients were in < 1-year age group (Fig. 3). Meningitis patients were younger compared to children with other predisposing factors (median, 0.46 versus 11.55 years, $p<0.001$ ) and sinusitis patients were older compared to children with other predisposing factors (median, 12.47 versus 2.64 years, $\mathrm{p}<0.001$ ). Other predisposing factors were not associated with age.

\section{Presenting symptoms}

For 89 patients symptom duration was documented and the median duration was 7 days (IQR 5-14 days). Most common symptoms were history of fever (66.7\%), vomiting $(66.7 \%)$, and headache $(58.1 \%)$ (Table 2$)$. The classical triad combining these symptoms was present in 13 patients $(14.0 \%)$.

\section{Microbiology}

60/93 patients $(74.2 \%)$ had blood cultures taken before antibiotic treatment and 17 were positive (28.3\%). CSF cultures were taken in $38 / 93$ patients (40.8\%) and 18 were positive $(47.4 \%)$. Intracranial pus was cultured in $70 / 93$ patients $(75.3 \%)$ and positive for 52 (74.3\%). Paranasal sinus cultures were taken from 23/93 patients (24.7\%) and positive in 14 (60.9\%). Overall 114 isolates of 53 species were grown amongst 69 patients (Fig. 4). 50/114 isolates were Streptococcus spp., of which Streptococcus milleri group organisms and Streptococcus pneumoniae were most often isolated, 25 and 8 times respectively. Streptococcus pyogenes was isolated 5 times.

16/114 isolates were Staphyloccus spp., of which 9 Staphyloccus aureus, and 7 coagulase-negative Staphylococci. Coagulase-negative Staphylococci were only considered relevant if isolated from intracranial pus culture.

6 culture-negative patients had PCR-positive samples for either Streptococcus intermedius, Streptococcus pneumoniae, Fusobacterium nucleatum, Neisseria meningitidis or Aspergillus spp. Additionally, 4 patients had microscopically positive samples for gram-positive cocci. In 13 patients all microbiological investigations were negative.

In 21 patients $(22.6 \%)$ multiple microorganisms were isolated from different sites (range, 2-6 microorganisms) and 12 patients (12.9\%) had polymicrobial abscess or empyema (range, 2-6 microorganisms). 21 anaerobic species were isolated from 12 patients, most commonly 


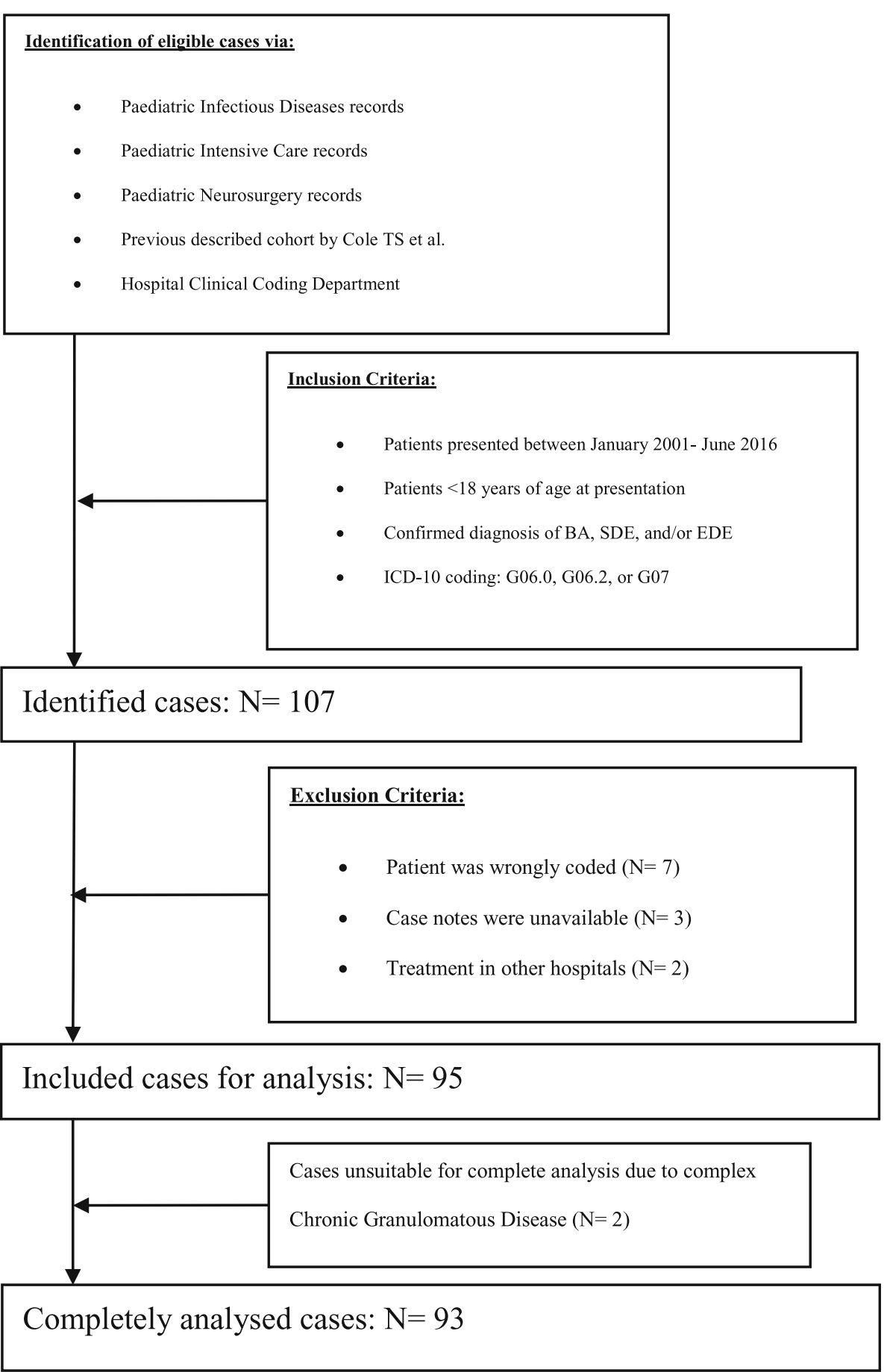

Fig. 1 Flowchart of Patient Identification

Prevotella spp. Anaerobic microorganisms were only isolated from children aged $1-15$ years.

\section{Antibiotic treatment}

92 patients were started on antibiotics and 3 on antifungals. 90 patients were started on empirical antibiotic regimens and two on quadruple therapy for tuberculosis.
Empirical antibiotic regimens consisted of 1-4 antibiotics and all but tuberculostatics were initially administered intravenously. 17 different antibiotics were given in 31 empirical regimens.

Most commonly administered empirical regimens consisted of a third-generation cephalosporin, metronidazole and amoxicillin in 29/90 patients (32.2\%). 7 patients 


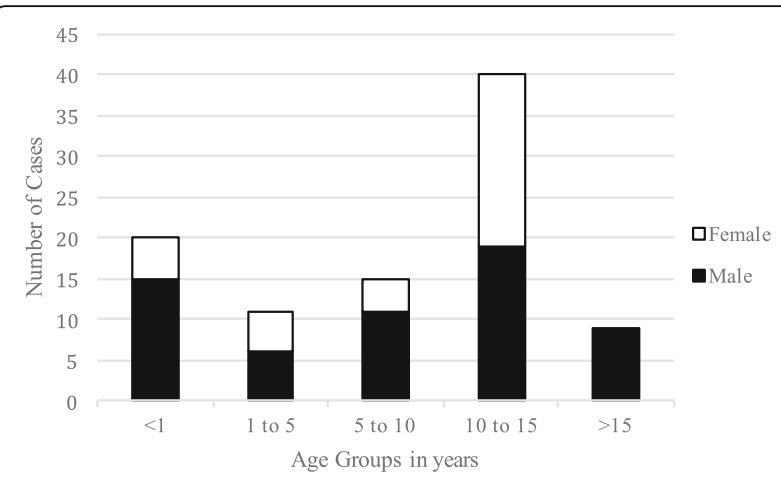

Fig. 2 Case Distribution in Age Groups

had a third-generation cephalosporin alone, 15 had a third-generation cephalosporin plus metronidazole, and 13 a third-generation cephalosporin plus metronidazole and a third antibiotic other than amoxicillin.

Antibiotic changes occurred in 86 patients (95.6\%). Reasons documented included microbiology results, antibiotic sensitivities, adverse reactions, and conversion to oral administration. 13 patients (14.4\%) developed antibiotic-related rashes and 6 antibiotic-related neutropenia. 18 patients required additional teicoplanin for presumed central line infections.

61 patients were converted to oral antibiotics (66.3\%) and 5 children received intrathecal antibiotics. Most oral regimens consisted of amoxicillin, amoxicillin/clavulanic acid, and/or metronidazole. Overall, 22 different antibiotics were used in this cohort.

Total duration of antibiotic treatment was documented for 89 patients, intravenous duration for 90 and oral treatment for 61 . For 3 patients, antibiotic treatment duration was unclear; 1 had an unclear end of intravenous treatment, and 2 an unclear end of oral treatment.

6 patients were excluded from antibiotic treatment duration analysis; 2 had Mycobacterium turberculosis and 2 had Actinomyces spp. infection, both known to require prolonged antibiotic treatment, one had complex Clostridium septicum infection requiring individualised prolonged antibiotic treatment and one patient died 9 days into treatment, not completing the intended antibiotic course.

Median total duration of antibiotic treatment was 92 days (IQR, 59-119 days), median duration of intravenous antibiotics 46.50 days (IQR, 25-71.50 days), and median duration of oral antibiotics 47 days (IQR, 38-77 days).

\section{Antibiotic coverage}

Sixty-nine patients on empirical antibiotics had growthpositive cultures. For $63 / 69$ patients full sensitivities were available and antibiotic coverage was analysed.

Chosen empirical regimens provided sufficient coverage in 60/63 patients (95.2\%). 2 patients had resistant microorganisms; one a metronidazole-resistant Dialister pneumosintes, and one an amoxicillin and cefotaxime-resistant Ochrobactrum anthropi. 31/63 patients (49.2\%) had an empirical regimen containing a third-generation cephalosporin or carbapenem plus amoxicillin, of which 30 did not require amoxicillin for additional coverage. One patient for whom amoxicillin provided necessary coverage grew a cefotaxime-resistant, amoxicillin-sensitive Enterococcus faecalis. The combination of a third-generation cephalosporin plus metronidazole would have been sufficient in $57 / 63$ patients (90.5\%), and a third-generation cephalosporin alone in 51/63 (81.0\%). Meropenem plus linezolid would have covered 60/63 patients (95.2\%). Linezolid was analysed rather than vancomycin because of superior CNS penetration which would be preferred despite potential side effects of linezolid (bone marrow suppression and neuropathy), as these are usually not observed in the first four weeks of treatment [25].

\section{Neurosurgery}

75/93 patients required neurosurgery (80.6\%). 35 patients underwent craniotomy, 30 burr hole drainage, 4 craniectomy, 4 aspiration via fontanelle, and 2 stereotactic aspiration. Median duration to neurosurgery was one day from admission (IQR 0-3 days). 30 patients required repeated neurosurgical interventions (maximum 6).

Table 1 Predisposing Factors $(N=95)$

\begin{tabular}{llll}
\hline Predisposing Factor & $\mathrm{N}(\%)$ & Predisposing Factor & $\mathrm{N}(\%)$ \\
\hline Sinusitis & $40(42.1 \%)$ & Immunocompromised & $4(4.2 \%)$ \\
Meningitis & $22(23.2 \%)$ & Congenital Brain Cyst & $2(2.1 \%)$ \\
Mastoiditis & $11(11.6 \%)$ & Congenital Heart Disease & $1(1.1 \%)$ \\
Otitis media & $7(7.4 \%)$ & Dental Infection & $1(1.1 \%)$ \\
Previous neurosurgery & $5(5.3 \%)$ & Haemolytic Uraemic Syndrome & $1(1.1 \%)$ \\
Distant Infection & $5(5.3 \%)$ & None Identified & $9(9.5 \%)$ \\
\hline
\end{tabular}




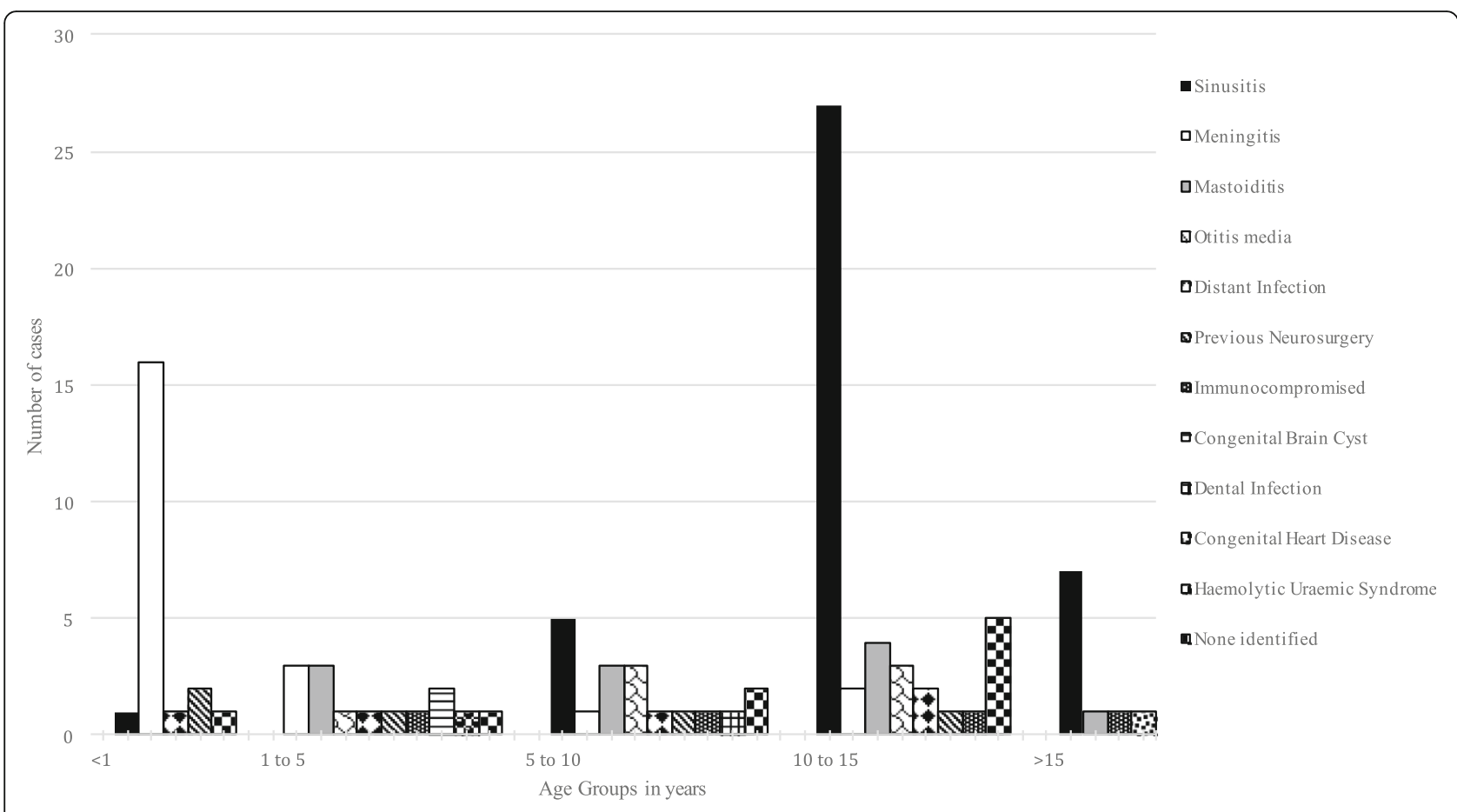

Fig. 3 Predisposing Factors by Age Group

\section{Outcome}

Mortality in this cohort was 3.2\%. Median duration of hospitalisation was 23 days (IQR, 15.5-34.5 days). Children exclusively treated with intravenous antibiotics did not require longer hospitalisation than children converted to oral antibiotics (median 25 versus 22 days, $p=0.266$ ).

Follow-up data was available for $87 / 92$ surviving patients $(93.4 \%)$. One patient was lost to follow-up due to emigration and 4 had an unclear follow-up duration. 10 patients were still in follow-up. For patients with completed follow-up the median duration was 7.99 months (IQR 4.92-12.64 months). Recurrence of intracranial suppurative infection occurred once in a child who developed bone flap osteomyelitis post-antibiotic treatment.

Thirty-five children (38.0\%) had short-term neurological sequelae $(<6$ months post-discharge) not present at initial presentation and 22 children (23.9\%) long-term neurological sequelae ( $\geq 6$ months post-discharge). Most common were seizures and hemiplegia (Table 3). Fifteen patients resolved short-term neurological sequelae within 6 months and 2 developed neurological sequelae $\geq 6$ months post-discharge.

Median symptom duration before admission was shorter for children with short-term neurological sequelae (6 versus 10 days, $p=0.002$ ) and with long-term neurological sequelae (5 versus 8.50 days, $p=0.037$ ), compared to patients without neurological sequelae. Initial Glascow Coma Scale (GCS) was not recorded for all patients, prohibiting meaningful statistical analysis. Although there seemed to be a trend for patients with a low GCS to have worse outcomes, also patients with maximal GCS, but who deteriorated rapidly after admission tended to have worse outcomes.

Table 2 Presenting Symptoms $(N=93)$

\begin{tabular}{llll}
\hline Symptom & $\mathrm{N}(\%)$ & Symptom & $\mathrm{N}(\%)$ \\
\hline Fever (History) & $62(66.7 \%)$ & Nuchal Rigidity & $14(15.1 \%)$ \\
Vomiting & $62(66.7 \%)$ & Rhinorrhoea & $13(14.0 \%)$ \\
Headache & $54(58.1 \%)$ & Papilloedema & $11(11.8 \%)$ \\
Focal Neurological Deficit & $35(37.6 \%)$ & Photophobia & $10(10.8 \%)$ \\
Fever $\left(>38.0^{\circ} \mathrm{C}\right)$ & $33(35.5 \%)$ & Nausea & $7(7.5 \%)$ \\
Lethargy & $33(35.5 \%)$ & Increased Head Circumference & $5(5.4 \%)$ \\
Altered Level of Consciousness & $27(29.0 \%)$ & Behavioural Change & $5(5.4 \%)$ \\
Seizure & $22(23.7 \%)$ & & \\
\hline
\end{tabular}




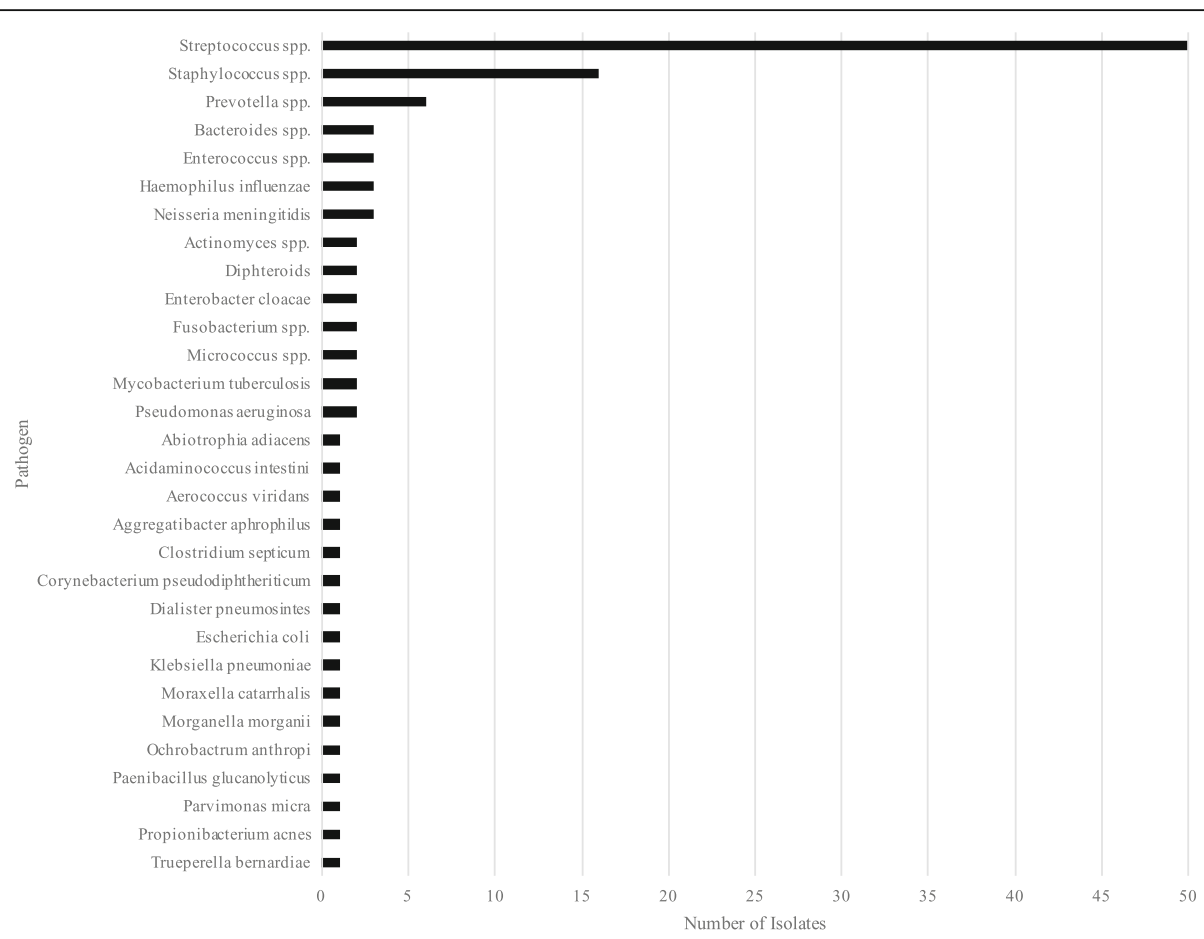

Fig. 4 Distribution of Isolated Pathogens in Growth-Positive Cultures $(N=114)$

Conversion to oral antibiotics was not associated with development of short- $(p=0.959)$ or long-term $(p=$ $0.135)$ neurological sequelae, compared to children exclusively on intravenous antibiotics.

Patients with unusual clinical courses or where S. pneumoniae was cultured were investigated for immunodeficiencies.
Only one patient with underlying immunodeficiency was identified.

\section{Discussion}

Our study is the largest single-centre study assessing local management of paediatric focal intracranial suppurative

Table 3 Neurological Sequelae $<6$ months and $\geq 6$ months post-discharge not present at admission

\begin{tabular}{|c|c|c|c|c|c|}
\hline $\begin{array}{l}\text { Neurological } \\
\text { sequelae }\end{array}$ & $\begin{array}{l}<6 \text { months post- } \\
\text { discharge }(n=92)\end{array}$ & $\begin{array}{l}\geq 6 \text { months post- } \\
\text { discharge }(n=92)\end{array}$ & $\begin{array}{l}\text { Neurological } \\
\text { sequelae }\end{array}$ & $\begin{array}{l}<6 \text { months post- } \\
\text { discharge }(n=92)\end{array}$ & $\begin{array}{l}\geq 6 \text { months post- } \\
\text { discharge }(n=92)\end{array}$ \\
\hline Any sequelae & 35 (38.0\%) & $22(23.9 \%)$ & Any sequelae & 35 (38.0\%) & $22(23.9 \%)$ \\
\hline Seizures & $10(28.6 \%)$ & $11(50 \%)$ & Nystagmus & $2(5.7 \%)$ & $2(9.1 \%)$ \\
\hline Hemiplegia & $6(17.1 \%)$ & $4(18.1 \%)$ & Torticollis & $2(5.7 \%)$ & - \\
\hline Diplopia & $4(11.4 \%)$ & $3(13.6 \%)$ & Dysdiadochokinesis & $1(2.9 \%)$ & - \\
\hline n.VII palsy & $4(11.4 \%)$ & $3(13.6 \%)$ & Hearing loss & $1(2.9 \%)$ & $2(9.1 \%)$ \\
\hline Speech abnormality & $4(11.4 \%)$ & $1(4.5 \%)$ & Hemianopia & $1(2.9 \%)$ & $2(9.1 \%)$ \\
\hline Gait abnormality & $3(8.6 \%)$ & $3(13.6 \%)$ & $\begin{array}{l}\text { Neurodevelopmental } \\
\text { delay }\end{array}$ & $1(2.9 \%)$ & $1(4.5 \%)$ \\
\hline Dysphasia & $3(8.6 \%)$ & $3(13.6 \%)$ & Memory difficulties & $1(2.9 \%)$ & $2(9.1 \%)$ \\
\hline $\begin{array}{l}\text { Delayed motor } \\
\text { development }\end{array}$ & $2(5.7 \%)$ & - & $\begin{array}{l}\text { Visual discrimination } \\
\text { difficulty }\end{array}$ & 1 (2.9\%) & - \\
\hline Hemiparesis & $2(5.7 \%)$ & $2(9.1 \%)$ & Learning difficulties & - & $2(9.1 \%)$ \\
\hline Hydrocephalus & $2(5.7 \%)$ & $3(13.6 \%)$ & $\begin{array}{l}\text { Concentration } \\
\text { difficulties }\end{array}$ & - & $1(4.5 \%)$ \\
\hline n. VI palsy & $2(5.7 \%)$ & - & Cerebral palsy & - & $1(4.5 \%)$ \\
\hline Neuropathic pain & $2(5.7 \%)$ & $1(4.5 \%)$ & & & \\
\hline
\end{tabular}


infection in order to analyse effectiveness of local management, identify causative pathogens and guide empirical antibiotic therapy. Our findings extended on Cole et al. [9] and demonstrated similarities and differences compared to case-series by other centres.

Our estimated annual incidence was higher than the $5.3 / 1,000,000$ previously reported in the UK. [12] The annual incidence in this tertiary centre was three times higher than in other developed countries. $[3,11,16,18$, $20,21]$ Our local annual incidence showed similarity with a Cameroonian study. [17] Reasons for this may lie in high rates for non-specific symptoms and subsequent lower suspicion for focal intracranial suppuration, and less health-seeking behaviour for upper respiratory tract infections. Median age at admission and bimodal age distribution are consistent with other studies. [12, 17]

Mortality in this cohort was $3.2 \%$, which is at the lower end of the spectrum previously reported (2.621.4\%). [3, 7, 11, 12, 15-18, 20-22] Neurological morbidity remains a significant problem, yet rates concur with other centres. [11, 12, 17, 18, 31] Long-term neurological sequelae affected a smaller proportion than short-term neurological sequelae, but might be underreported as follow-up in tertiary care ended, and neuropsychological effects and mild cognitive impairments are difficult to diagnose.

Predisposing factors were identified in $90.5 \%$. Meningitis was associated with younger and sinusitis with older age, which is unsurprising given sinus maturation. High rates of sinusitis and meningitis may be explained by the number of SDE patients in this cohort. There is an association between SDE and meningitis and sinusitis, previously demonstrated by Legrand et al. [16] Adolescent patients are more at risk for intracranial complications of sinusitis compared to younger children and adults, and sinusitis patients were mostly adolescents. [32] Adolescents are more likely to develop intracranial extension of sinusitis, as vascularity and blood supply of diploic veins is known to be increased compared to adults. [33] $\mathrm{CHD}$ as a predisposing factor in this study was rare. This has been reported by other centres [3, 17, 31], although some centres report $\mathrm{CHD}$ as their main predisposing factor in $20.8-40 \%$. [7, 18, 20, 21]

$35.6 \%$ had focal neurological deficits at presentation and $23.7 \%$ seizures at admission. This is similar to other centres $[11,17,18]$, although rates go up to $53 \%$ [12] and $48 \%$ [21] respectively. In this study the most common symptoms were non-specific. The classic triad was seldom seen. This is in accordance to literature from developed countries $[3,18]$, while developing countries see the triad in up to $52 \%$ [17]. Children with poorer outcome had shorter symptom duration, and tended to have more severe altered levels of consciousness, which may be attributed to more rapid deterioration. Although otorhinolaryngological infections were prevalent in this cohort, concurrent symptoms were not often reported and may be underreported since patients often had more severe presenting clinical features.

Streptococci were most commonly isolated with Streptococcus milleri group organisms as most common species. The second largest group were Staphylococci, although it is debatable how many coagulase-negative isolates were pathogenic. The predominance of Streptococci has been described previously in the UK [12], as well as in other countries $[3,7,11,16,20-22,31] .12 .9 \%$ of isolates were anaerobic which concurs with published literature. [11, 12] Interestingly Prevotella spp. were most common, whereas other centres report Fusobacterium spp. as the most common anaerobic isolate. $[3,11,20]$

This study showed great variability in empirical regimens and in antibiotic treatment duration. This has been noted previously $[3,11,12]$, and demonstrates the lack of uniform guidelines and potentially a change in practice over the 15 years covered by the study. Nonetheless, chosen regimens provided sufficient coverage in the majority of patients. Broad-spectrum antibiotics remain necessary before microbiology results, as gram-positive, gram-negative and anaerobe microorganisms were all cultured in this cohort. Combinations including third-generation cephalosporins plus metronidazole were successful in the majority of patients. $90.5 \%$ of patients with growth-positive cultures would have been sufficiently covered with a third-generation cephalosporin plus metronidazole, and most patients with negative cultures improved clinically on this regime. Therefore, we recommend this regime as first-choice empirical antibiotic treatment. Despite meropenem plus linezolid covering a greater proportion, this regimen should be reserved for severe or treatment non-responsive cases. Over the 15.5-year period, there have not been any anaerobic pathogens cultured in the $<1$ and $>15$-years age groups. In infants, the routine use of metronidazole might be reconsidered if anaerobic cultures are negative. The $>15$-years age group was too small to make a similar recommendation.

\section{Strengths and limitations}

This study has the largest number of paediatric patients in a single-centre cohort. This enabled us to comprehensively review the management of paediatric focal intracranial suppurative infections.

Limitations lie in the retrospective nature of the study, leading to potential information bias. $2.8 \%$ of cases had to be excluded because of unavailable case notes. Long-term complications possibly have been underreported as follow-up ended and patients may have presented with sequelae to local hospitals or have subtler, underrecognised, neuropsychological sequelae. The single-centre aspect of this study makes results difficult to generalise, and there are differences compared to 
other single-centre studies. However, it shares similarities with other centres and provides additional data to assist clinical choices in centres with similar microbiological profiles and facilities.

In conclusion, paediatric focal intracranial suppurative infection continues to cause significant mortality and morbidity. Although uncommon, it occurs more frequently in the North East of England than previously reported in developed countries. Empirical antibiotic regimens should provide broad-spectrum coverage and we recommend a third-generation cephalosporin plus metronidazole. Meropenem and linezolid should be reserved for severe and complex cases. In infants the use of metronidazole might be reconsidered if the microbiology results are negative for anaerobes. Optimum duration of antibiotic treatment remains unclear. A multi-centred, prospective randomised controlled trial would be required to answer this question. Further research should focus on the identification of factors causing the local higher occurrence paediatric focal intracranial suppurative infection and the development of guidelines for antibiotic treatment.

\section{Abbreviations}

BA: brain abscess; CGD: chronic granulomatous disease; CHD: congenital heart disease; CRP: C-reactive protein; CSF: Cerebrospinal fluid;

EDE: extradural empyema; GCS: Glasgow Coma Scale; GNCH: Great North Children's Hospital; IQR: interquartile range; PCR: polymerase chain-reaction; PICU: Paediatric Intensive Care Unit; SDE: subdural empyema; UK: United Kingdom

\section{Acknowledgements}

Not applicable.

\section{Funding}

There were no sources of funding for this research report.

\section{Availability of data and materials}

The datasets used and/or analysed during the current study are available from the corresponding author on reasonable request.

\section{Authors 'contributions \\ FV performed the study as main researcher and collected and analysed the patient data. He combined all data in the database and performed the statistical analysis. He was the main writer of this manuscript. AB was a major contributor in writing the manuscript and supervising FV in the statistical process. LPC analysed microbiological profiles and corresponding antibiotic and helped in writing the microbiological sections. NR helped identifying and analysing the neurosurgical interventions performed on the patients. SB analysed MRIs and CTs of patients suspected to have focal intracranial suppurative infection due to sinusitis. ME supervised the research, guided FV, checked the statistical analysis and reviewed antibiotic schemes. She was a major contributor in writing the manuscript. All authors read and approved the final manuscript.}

\section{Ethics approval and consent to participate}

Local ethical approval and consent for the use of human data was obtained by de Caldicott commission for the Newcastle upon Tyne Hospitals NHS Foundation Trust Reference number: ID: 4738.

\section{Consent for publication}

Not applicable.

\section{Competing interests}

The authors declare that they have no competing interests.

\section{Publisher's Note}

Springer Nature remains neutral with regard to jurisdictional claims in published maps and institutional affiliations.

\section{Author details}

${ }^{1}$ Paediatric Immunology, Infectious Diseases and Allergy Department, Newcastle upon Tyne Hospitals NHS Foundation Trust, Great North Children's Hospital, Newcastle upon Tyne NE1 4LP, UK. ²Erasmus MC, Rotterdam 3015,

CE, The Netherlands. ${ }^{3}$ Microbiology Department, Newcastle upon Tyne Hospitals NHS Foundation Trust, Royal Victoria Infirmary, Newcastle upon Tyne NE1 4LP, UK. ${ }^{4}$ Neurosurgery department, Newcastle upon Tyne Hospitals NHS Foundation Trust, Royal Victoria Infirmary, Newcastle upon Tyne NE1 4LP, UK. ${ }^{5}$ Otorhinolaryngology, Newcastle upon Tyne Hospitals NHS Foundation Trust, Royal Victoria Infirmary, Newcastle upon Tyne NE1 4LP, UK. ${ }^{6}$ Institute of Cellular Medicine, Newcastle University, Newcastle upon Tyne NE2 4HH, UK.

Received: 1 May 2018 Accepted: 3 April 2019

Published online: 25 April 2019

\section{References}

1. Bonfield CM, Sharma J, Dobson S. Pediatric intracranial abscesses. J Inf Secur. 2015;71(Suppl 1):S42-6.

2. Frazier $\mathrm{L}$, Ahn ES, Jallo Gl. Management of brain abscesses in children. Neurosurg Focus. 2008;24(6):E8.

3. Shachor-Meyouhas Y, Bar-Joseph G, Guilburd JN, Lorber A, Hadash A, Kassis I. Brain abscess in children - epidemiology, predisposing factors and management in the modern medicine era. Acta Paediatr. 2010;99(8):1163-7.

4. Sheehan JP, Jane JA, Ray DK, Goodkin HP. Brain abscess in children Neurosurg Focus. 2008;24(6):E6.

5. Saez-Llorens X. Brain abscess in children. Semin Pediatr Infect Dis. 2003; 14(2):108-14.

6. Bockova J, Rigamonti D. Intracranial empyema. Pediatr Infect Dis J. 2000; 19(8):735-7.

7. Ozsurekci Y, Kara A, Cengiz AB, Celik M, Ozkaya-Parlakay A, Karadag-Oncel E, Ceyhan M. Brain abscess in childhood: a 28-year experience. Turk J Pediatr. 2012:54(2):144-9.

8. Yogev R, Bar-Meir M. Management of brain abscesses in children. Pediatr Infect Dis J. 2004;23(2):157-9.

9. Cole TS, Clark JE. Paediatric focal intracranial suppuration: a UK single-Centre experience. Childs Nerv Syst. 2013;29(2):185.

10. Goodkin HP, Harper MB, Pomeroy SL. Intracerebral abscess in children: historical trends at Children's hospital Boston. Pediatrics. 2004;113(6): 1765-70.

11. Gelabert-Gonzalez M, Serramito-Garcia R, Garcia-Allut A, Cutrin-Prieto J. Management of brain abscess in children. J Paediatr Child Health. 2008. 44(12):731-5.

12. Felsenstein S, Williams B, Shingadia D, et al. Clinical and microbiologic features guiding treatment recommendations for brain abscesses in children. Pediatr Infect Dis J. 2013;32(2):129-35.

13. Sennaroglu L, Sozeri B. Otogenic brain abscess: review of 41 cases. Otolaryngol Head Neck Surg. 2000;123(6):751-5.

14. Brouwer MC, Coutinho JM, van de Beek D. Clinical characteristics and outcome of brain abscess: systematic review and meta-analysis. Neurology. 2014;82(9):806-13.

15. Madhugiri VS, Sastri BV, Srikantha U, et al. Focal intradural brain infections in children: an analysis of management and outcome. Pediatr Neurosurg. 2011;47(2):113-24

16. Legrand M, Roujeau T, Meyer P, Carli P, Orliaguet G, Blanot S. Paediatric intracranial empyema: differences according to age. Eur J Pediatr. 2009; 168(10):1235-41.

17. Djientcheu VP, Mouafo TF, Esiene A, et al. Intracranial suppurations in the African child: a severe but preventable complication. Childs Nerv Syst. 2013; 29(1):119-23.

18. Canpolat M, Ceylan O, Per H, et al. Brain abscesses in children: results of 24 children from a reference center in Central Anatolia, Turkey. J Child Neurol. 2015;30(4):458-67. 
19. Brouwer MC, Tunkel AR, McKhann GM 2nd, van de Beek D. Brain abscess. N Engl J Med. 2014;371(5):447-56.

20. Laulajainen-Hongisto A, Lempinen L, Farkkila E, et al. Intracranial abscesses over the last four decades; changes in aetiology, diagnostics, treatment and outcome. Infect Dis (Lond). 2015:1-7.

21. Lee CG, Kang SH, Kim YJ, Shin HJ, Choi HS, Lee JH, Lee MH. Brain abscess in Korean children: a 15-year single center study. Korean J Pediatr. 2010;53(5): 648-52.

22. Leotta N, Chaseling R, Duncan G, Isaacs D. Intracranial suppuration. J Paediatr Child Health. 2005;41(9-10):508-12.

23. Muzumdar D, Jhawar S, Goel A. Brain abscess: an overview. Int J Surg. 2011; 9(2):136-44

24. Arlotti M, Grossi P, Pea F, et al. Consensus document on controversial issues for the treatment of infections of the central nervous system: bacterial brain abscesses. Int J Infect Dis. 2010;14 Suppl 4:S79-92.

25. Nau R, Sorgel F, Eiffert H. Penetration of drugs through the bloodcerebrospinal fluid/blood-brain barrier for treatment of central nervous system infections. Clin Microbiol Rev. 2010;23(4):858-83.

26. Ratnaike TE, Das S, Gregson BA, Mendelow AD. A review of brain abscess surgical treatment--78 years: aspiration versus excision. World Neurosurg. 2011;76(5):431-6.

27. The Infection in Neurosugery Working Party of the British Society for Antimicrobial Chemotherapy. The rational use of antibiotics in the treatment of brain abscess. Br J Neurosurg. 2000;14(6):525-30

28. World Health Organization. ICD version 2016 Available at: http://apps.who. int/classifications/icd10/browse/2016/en. Accessed 28 Aug 2016.

29. Public Health England. Child Health Profile: Newcastle upon Tyne 2016 Available at: https://fingertips.phe.org.uk/profile/child-health-profiles/areasearch-results/E08000021?place_name=Newcastle\%20upon\%20Tyne\&search_ type=parent-area. Accessed 15 Apr 2019 .

30. Public Health England. Child Health Profile: Cumbria 2016 Available at: https://fingertips.phe.org.uk/profile/child-health-profiles/area-search-results/ E10000006? place_name=Cumbria\&search_type=parent-area. Accessed 15 Apr 2019.

31. Raffaldi I, Garazzino S, Castelli Gattinara G, et al. Brain abscesses in children: an Italian multicentre study. Epidemiol Infect. 2017;145(13):2848-55.

32. Germiller JA, Monin DL, Sparano AM, Tom LW. Intracranial complications of sinusitis in children and adolescents and their outcomes. Arch Otolaryngol Head Neck Surg. 2006;132(9):969-76.

33. Nicoli TK, Oinas M, Niemela M, Makitie AA, Atula T. Intracranial Suppurative complications of sinusitis. Scand J Surg. 2016.

Ready to submit your research? Choose BMC and benefit from:

- fast, convenient online submission

- thorough peer review by experienced researchers in your field

- rapid publication on acceptance

- support for research data, including large and complex data types

- gold Open Access which fosters wider collaboration and increased citations

- maximum visibility for your research: over $100 \mathrm{M}$ website views per year

At $\mathrm{BMC}$, research is always in progress.

Learn more biomedcentral.com/submissions 